\title{
Indicadores utilizados en el aprendizaje virtual de Institutos Superiores Técnicos y Tecnológicos en Ecuador
}

José Luis Vera Solórzano joseluisverasol@gmail.com

Instituto Superior Tecnológico Atlantic

Hugo Ángel Quintero Bravo

hugo.quintero@uleam.edu.ec

Universidad Laica Eloy Alfaro de Manabi. ULEAM

Victor Rene Gracia Peña

sercomgar@gmail.com

\section{Unidad Educativa Siglo XXI Eladio Roldos}

\section{Resumen}

El presente artículo tiene como objetivo estudiar la existencia de indicadores utilizados en el aprendizaje virtual de los Institutos Superiores Técnicos y Tecnológicos (ISTT) en Ecuador, tomando en cuenta que la modalidad virtual es una alternativa para acceder a la educación superior. Para ello fue necesario revisar los procesos de evaluación a los que han sido sometidos los ISTT. El diseño metodológico utilizado fue de tipo documental. Se revisaron varios trabajos e informes de evaluación realizados por los organismos de control; entre los principales resultados obtenidos se evidenció que la formación de nivel técnica y tecnológica no ofrece carreras en modalidad virtual y por consiguiente los docentes han adquirido las destrezas tecnológicas requeridas; se concluyó que los Institutos Superiores Técnicos y Tecnológicos no han desarrollado una cultura de calidad en función de los parámetros establecidos, solo se trata de cumplir las exigencias del CACES por obligación, mas no por vocación, en este sentido al no existir indicadores sobre el aprendizaje virtual en este nivel de formación, no se han realizado los esfuerzos necesarios para promover la modalidad virtual como alternativa de accesibilidad y cubrir la demanda insatisfecha de los estudiantes.

\section{Palabras Clave.}

Evaluación, Indicadores, Aprendizaje Virtual, Institutos Superiores

Recibido 08/09/2019 - 17/10/2019 


\title{
Indicators used in virtual learning of Higher Technical and Technological Institutes in Ecuador
}

\begin{abstract}
This article aims to study the existence of indicators used in virtual learning of a Higher Technical and Technological Institutes (HTTI) in Ecuador, taking into account that virtual modality is an alternative to access higher education. For this, it was necessary to review the evaluation processes to which the HTTIs have been submitted. The methodological design used was documentary. Several papers and evaluation reports made by the control agencies were reviewed; Among the main results obtained, it was evidenced that technical and technological level training does not offer careers in virtual mode and therefore teachers have acquired the required technological skills; it was concluded that the Higher Technical and Technological Institutes have not developed a culture of quality based on the established parameters, it is only about complying with the requirements of CACES by obligation, but not by vocation, in this sense as there are no indicators on learning virtual at this level of training, no efforts have been made to promote the virtual modality as an accessibility alternative and meet the unsatisfied demand of the students.
\end{abstract}

\section{Keywords.}

Evaluation, Indicators, Virtual Learning, Higher Institutes 


\section{Indicadores utilizados na aprendizagem virtual dos Institutos Técnicos e Tecnológicos Superiores no Equador}

\section{Resumo}

Este artigo tem como objetivo estudar a existência de indicadores utilizados na aprendizagem virtual dos Institutos Técnicos e Tecnológicos Superiores (ISTT) no Equador, considerando que a modalidade virtual é uma alternativa para acessar o ensino superior. Para isso, foi necessário revisar os processos de avaliação aos quais os ISTTs foram submetidos. O desenho metodológico utilizado foi documental. Vários artigos e relatórios de avaliação elaborados pelas agências de controle foram revisados; Dentre os principais resultados obtidos, constatou-se que o treinamento em nível técnico e tecnológico não oferece carreiras em modo virtual e, portanto, os professores adquiriram as habilidades tecnológicas necessárias; Concluiu-se que os Institutos Técnicos e Tecnológicos Superiores não desenvolveram uma cultura de qualidade com base nos parâmetros estabelecidos; trata-se apenas de cumprir os requisitos do CACES por obrigação, mas não por vocação, neste sentido, pois não há indicadores de aprendizagem. virtual neste nível de treinamento, não foram feitos esforços para promover a modalidade virtual como alternativa de acessibilidade e atender à demanda insatisfeita dos alunos.

\section{Palavras chave.}

Avaliação, Indicadores, Aprendizado Virtual, Institutos Superiores. 


\section{Introducción}

Las Tecnologías de la Información y Comunicación (TIC) han conquistado todos los sectores de la sociedad, hoy en día su utilización es imprescindible para casi todas las entidades ya sean públicas o privadas, sin importar la actividad que realicen o si ofrecen productos tangibles (bienes) o productos intangibles (servicios), requieren de las TIC para ser competitivos. En el ámbito educativo no se puede mirar para un costado y resistirse al cambio, al contrario, se debe evolucionar junto con el desarrollo de la ciencia y tecnología, al final de cuentas la educación es el medio que potencia el desarrollo de las sociedades a través de los productos y servicios que genera el conocimiento adquirido en su sistema educativo.

En este sentido es evidente que la educación ha dado un giro importante en la forma que interactúan docentes y estudiantes, si bien el principio fundamental de la educación es prácticamente el mismo cuanto a su finalidad, para nadie es un secreto que la tecnología ha invadido todos los sectores, el uso de dispositivos fijos y/o móviles sumado a las aplicaciones y servicios que brindan a los usuarios, ha generado un cambio radical en la forma de comunicación entre los principales operativos del sistema educativo, es decir entre el profesor y el estudiante.

Los entornos virtuales de aprendizaje han ganado mucho espacio en el sistema de educación media y más aún en el sistema de educación superior, ofertando carreras de grado y/o de cuarto nivel con la ayuda de plataformas virtuales. La calidad de los programas de educación universitaria virtual es un aspecto complejo, sobre todo, por su carácter multifactorial. Algunos autores relacionan dicha calidad con la "satisfacción de usuarios, excelencia del sistema, efectividad del proceso, resultados académicos positivos y buen impacto social del programa” (Fainholc, 2004, p. 2). Para Ruiz y Dávila (2016), "la calidad de la formación en la modalidad virtual es una función de las interacciones complejas que se producen entre los diferentes factores que intervienen en los resultados de aprendizaje para satisfacer necesidades personales y sociales” (p. 17). En la opinión de García (2014), cuando hablamos de calidad asumimos que nos queremos referir a la buena calidad, o a la excelencia, 
o a la eficacia en grado sumo. Así, si hablamos de buena calidad de educación a distancia nos deberíamos estar refiriendo a una educación a distancia eficaz y de calidad (positiva)" (p. 115-116).

En este estudio se aborda "los indicadores utilizados en el aprendizaje virtual de Institutos Superiores Técnicos y Tecnológicos en Ecuador”, para ello es necesario hacer un recorrido sobre este tipo de formación, sus perfiles profesionales, su mercado laboral, su rol en la sociedad y que indicadores deberían tomarse en las diferentes modalidades de estudio en este nivel de formación. A continuación, se describen los aspectos más importantes de la educación superior en el Ecuador, en especial en el nivel de formación técnica y tecnológica, que en los últimos años ha sufrido cambios importantes en beneficio de los estudiantes en cuanto al reconocimiento y revaloración social de estos estudios.

\section{Educación técnica y tecnológica en Ecuador}

La educación técnica en Ecuador tiene sus orígenes a finales del siglo XIX, con la creación de la Escuela de Artes y Oficios del Protectorado Católico, con la finalidad de formar a los obreros artesanales e industriales y darles una instrucción técnica y estética. Con el paso de los años se convirtió en el Colegio Central Técnico de Quito (CEAACES, 2013). La formación técnica se fortaleció a partir del inicio del auge petrolero en los años 70 del siglo XX, que generó los recursos necesarios para la inversión en el área educativa y a la vez incrementó la demanda de mano de obra calificada (Montoya, Rossi y Samaniego, 1988 citado en (CEAACES, 2013). Con la Ley de Educación y Cultura de 1977, se crea el "postciclo diversificado" o post-bachillerato" con dos años de duración y tres grandes ramas: técnica y tecnológica, artes y pedagogía, que se desarrollaban, respectivamente, en los Institutos Técnicos Superiores, los Institutos de Artes y los Institutos Normales.

Aunque estos procesos educativos superaban el nivel medio de enseñanza, las instituciones en las que se desarrollaban, funcionaban en las propias instalaciones de los centros de enseñanza de nivel medio que les dieron origen. Así mismo, eran reguladas por el propio Ministerio de Educación y Cultura, con asesoría del Consejo Nacional de Educación y Cultura (Congreso Nacional del Ecuador, 1977). 
A partir de la Constitución de 1998, la Educación Técnica y Tecnológica posterior al bachillerato pasó a formar parte del Sistema de Educación Superior. Esa carta magna dispuso que los Institutos Superiores Técnicos y Tecnológicos (ISTT) fueran regulados por el Consejo Nacional de Educación Superior - CONESUP, definido en la constitución como el órgano de control, regulación y planificación del sistema de educación superior (Asamblea Constituyente del Ecuador, 1998) y cuya composición y funciones se definieron en la Ley de Educación Superior del año 2000. Sin embargo, el cambio de organismo regulatorio no modificó el hecho de que los institutos superiores, principalmente los de régimen público, continuaron funcionando en las instalaciones y en el seno de la estructura administrativa y financiera de las instituciones de educación media de las que surgieron.

Como parte del proceso de fortalecimiento de la Educación Superior Técnica y Tecnológica, se inicia en 2011 la separación paulatina de los institutos superiores de las instituciones de nivel medio originarias, independencia que ha abarcado progresivamente los ámbitos académico, administrativo, financiero y de infraestructura (SENESCYTMINEDUC, 2011).

En 2013 el CEAACES, con base en la información reportada por las instituciones al Sistema Nacional de Información de Educación Superior del Ecuador, SNIESE, en 2010, realizó un estudio (CEAACES, 2013) de 184 ISTT que reportaron información completa al SNIESE.

\section{Indicadores Evaluación de los Institutos Superiores Técnicos y Tecnológicos}

La evaluación de los institutos en Ecuador ha pasado por tres evaluaciones, la primera en el año 2007, la segunda en el año 2009 y la tercera en el año 2014.

La primera evaluación institucional de los institutos superiores técnicos y tecnológicos de la que se tiene referencia fue iniciada por el antiguo Consejo Nacional de Evaluación y Acreditación de la Educación Superior Ecuatoriana (CONEA) en 2007. En aquel proceso evaluativo se partía de una autoevaluación institucional que era sucedida por la verificación de la misma con base en un modelo construido al efecto. El resultado de la evaluación se expresaba en la acreditación o no de la institución optante. 
Al modelo aplicado por el CONEA se le conceptualizó como sistémico y en él se identificaban cuatro funciones de la institución de educación superior: Docencia, Investigación, Vinculación con la comunidad y Gestión administrativa. A partir de las funciones se identificaron ocho ámbitos, al interior de cada cual "se realizó un análisis por medio de la verificación de indicadores para establecer el cumplimiento de 96 estándares”. La metodología consideraba a "los estándares como definiciones cualitativas y medibles que expresan objetivamente el nivel deseable contra el que se contrastan los indicadores (. . .)”. Un elemento peculiar de la propuesta metodológica fue que "se consideraron la totalidad de estándares con la misma importancia sin discriminar entre indispensables y necesarios" (CONEA, 2007a, p. 8).

Como se puede apreciar la primera evaluación estuvo basada en indicadores para medir el nivel de calidad en función de alcanzar un estándar establecido y así determinar el nivel de cumplimiento, el problema principal radicó en que no fue socializado adecuadamente el modelo de evaluación aplicado, las autoridades de los ISTT no tenían muy claro las evidencias de las cuatro funciones que debían presentar que debían presentar y la proporción de cada uno de ellos, sin embargo hay que reconocer que en su momento no se le dio la debida importancia.

La segunda evaluación de los institutos superiores estuvo orientada según el Mandato Constituyente 14, se desarrolló en 2009 y sus resultados fueron publicados en diciembre de ese año. Para ese proceso, basado en la aplicación de Métodos de Decisión Multicriterio (MDM), fue construido un modelo de evaluación genérico del que se derivaron particularidades, que fueron definidas en función del énfasis administrativo, industrial o agropecuario en el perfil académico de la institución. La evaluación realizada clasificó a los 245 Institutos Superiores Técnicos y Tecnológicos evaluados en tres categorías de desempeño institucional: A (70-100\% de cumplimiento), B (20-69\% de cumplimiento) y C (inferior al 20\% de cumplimiento) (CONEA, 2009a).

En esta evaluación se realizó función de tres criterios: Docentes, Estudiantes y Administración, es decir no se tomó en cuenta la investigación y la vinculación al menos 
desde la precepción literal de los criterios propuestos, esa fue la principal diferencia con la primera evaluación.

La tercera evaluación institucional para los Institutos Superiores Técnicos y Tecnológicos, se realizó en 2014, contando con cinco criterios que son: Pertinencia, Currículo, Calidad de la docencia, Infraestructura y Ambiente institucional. Cada uno de estos criterios consta de varios indicadores que en conjunto suman 50 indicadores de evaluación. (CEAACES, 2015).

Esta evaluación fue más estructurada en cuanto a forma y fondo, la gran mayoría de institutos ya habían pasado por dos evaluaciones anteriores y de alguna manera ya se tenía alguna idea de las evidencias que se debía presentar, sin embargo, se concentraron mucho en el producto que debía presentar y se olvidaron del proceso de construcción de esa evidencia, lo cual dejó al descubierto que no existe una cultura institucional para trabajar en función de la calidad.

Hasta el momento se ha hecho un recorrido de los procesos evaluativos a los que han sido sometidos los Institutos Superiores Técnicos y Tecnológicos en el Ecuador, ahora es preciso analizar las carreras en modalidad virtual a distancia y en línea con la finalidad de determinar los indicadores que permitan evaluar la calidad de esta oferta académica.

\section{Carreras en modalidad virtual a distancia y en línea en Ecuador}

Tomando en cuenta que la calidad de educación superior a distancia y virtual es uno de los ejes de análisis para universidades y organismos de control de educación superior a nivel mundial; en este sentido la UNESCO en una conferencia celebrada en París en el año 2009 manifestó que el aprendizaje abierto y a distancia apoyado del uso de las TIC permiten ampliar la oferta académica y diversificar las oportunidades de acceso a la educación superior de calidad.

La necesidad de garantizar el acceso de la educación superior para miles de jóvenes ha hecho que los órganos de control específicamente la Secretaría Nacional de Educación Superior Ciencia Tecnología e Innovación (SENESCYT) a través de sus autoridades presenten una nueva oferta de Educación Superior Virtual a distancia y en línea junto con cinco universidades del país como son la Universidad Técnica del Norte, Universidad Central 
del Ecuador, Universidad Estatal de Milagro, Universidad Técnica de Manabí y Universidad de las Fuerzas Armadas (ESPE), esta oferta incluye 10 carreras que son: Comunicación Social, Derecho, Economía, Educación Básica, Educación Inicial, Tecnologías de la Información, Pedagogía de los Idiomas, Psicología, Trabajo Social, Turismo.

Como se ha podido apreciar las ofertas en modalidad virtual a distancia y en línea aplican según la propuesta solo para universidades, en este caso las cinco universidades antes mencionadas, es decir que los institutos no han sido tomados en cuenta para ofrecer estas modalidades, la gran interrogante es saber si en un futuro se ampliará esta oferta para formación técnica y tecnológica, por el momento sigue siendo una incertidumbre hasta que se den las condiciones óptimas y los institutos estén preparados para ofrecer las modalidades antes mencionadas. De acuerdo con lo mostrado por EducarPlus (2018) hay una lista de 85 Institutos Superiores Técnicos y Tecnológicos autofinanciados, donde se puede apreciar entre otras cosas la ubicación y la modalidad de estudios, siendo esta última de interés para el presente estudio.

De los 85 institutos autofinanciados existentes en el país solo seis tienen dentro de su oferta académica carreras en modalidad a distancia, los demás tienen carreras en modalidad presencial y semipresencial. Es evidente que en este tipo de formación la modalidad virtual no está dentro de sus opciones al menos por el momento, las variables de escases en este tipo de ofertas pueden ser que no se cuenta con la infraestructura tecnológica necesaria o con las competencias tecnológicas por parte de los docentes.

\section{Programa formativo virtual y sus elementos}

|Aunque existen varias definiciones se tomará como referencia a Merciniak (2016) quien define a los programas virtuales como:

Un documento (informatizado o no) que identifica acciones orientadas a lograr objetivos formativos para un periodo determinado, y que incluye un conjunto de elementos necesarios para el desarrollo del proceso de enseñanza-aprendizaje de una asignatura determinada impartida en la modalidad virtual. Se realiza completamente mediante a las Tecnologías de la Información y Comunicación, con el soporte tecnológico de una plataforma educativa virtual y con apoyo de un/os docente/s en línea, quien/es orienta/n 
el desarrollo académico de los estudiantes utilizando diversas vías de comunicación sincrónica y asincrónica (Marciniak, 2016, p. 70).

La definición antes mencionada es muy completa por cuanto hace referencia a que debe estar orientada a alcanzar objetivos académicos al igual que la educación presencial, para ello es necesario contar con una plataforma virtual apropiada, pero sobre todo que los docentes posean las competencias tecnológicas necesarias.

En un documento elaborado por el Higher Education Committee on Quality (HECQ, 1997) titulado "Directrices para la Educación a Distancia" se encuentran algunos estándares académicos sugeridos para los programas no presenciales de educación superior, de igual manera se presentan algunas propuestas sobre qué elementos deberían tener estos programas para ofrecer un servicio educativo de buena calidad. Por ello, se espera que la universidad:

Tome en cuenta las habilidades, el conocimiento y la experiencia de los estudiantes matriculados y de las circunstancias en las cuales se espera que éstos hagan uso del sistema de distancia de la institución.

$\checkmark$ Explicite los principios básicos para seleccionar los medios de enseñanza elegidos.

$\checkmark$ Considere y especifique de forma precisa y detallada los recursos de aprendizaje y ayuda que serán puestos a disposición directa de los estudiantes por la institución proveedora.

$\checkmark$ Considere cómo conseguir que los materiales de aprendizaje sean interactivos y permitir la retroalimentación formativa de los estudiantes.

$\checkmark$ Detalle el horario de las actividades del estudiante en relación con el programa de estudio usando el sistema previsto para el aprendizaje a distancia. (HECQ, 1997, p.9-10). 
Lo antes expuesto permite deducir que un programa virtual debe incluir de manera imprescindible los siguientes elementos:

$\checkmark$ Metas de aprendizaje,

$\checkmark$ Competencias,

$\checkmark$ Estrategias de enseñanza,

$\checkmark$ Estrategias de evaluación del aprendizaje,

$\checkmark$ Perfil del estudiante,

$\checkmark$ Materiales educativos interactivos,

$\checkmark$ Recursos de aprendizaje,

$\checkmark$ Recursos del apoyo a los estudiantes,

$\checkmark$ Contenido temático,

$\checkmark$ Cronograma de actividades del estudiante.

En la siguiente tabla se presenta algunos elementos que debería tener un programa virtual según varios autores entendidos en el tema.

\begin{tabular}{lll}
\hline \multicolumn{1}{c}{ Autores } & \multicolumn{1}{c}{ Elementos del programa virtual } \\
& $\checkmark$ & Aspectos específicos del programa \\
& $\checkmark$ & Temas y objetivos \\
Meza (2012) & $\checkmark$ & Actividades de aprendizaje \\
& $\checkmark$ & Evaluación del aprendizaje \\
& $\checkmark$ & Tutoría virtual \\
\hline & $\checkmark$ & Necesidades de los estudiantes \\
& $\checkmark$ & Objetivos formativos \\
& $\checkmark$ & Competencias \\
& $\checkmark$ & Contenidos \\
& $\checkmark$ & Material didáctico \\
& $\checkmark$ & Actividades de aprendizaje \\
& $\checkmark$ & Evaluación del aprendizaje \\
& $\checkmark$ & Evaluación del programa \\
\hline
\end{tabular}




\begin{tabular}{|c|c|c|}
\hline \multirow{6}{*}{ Shattuck (2014) } & $\checkmark$ & Aspectos específicos del programa (nombre, duración, justificación) \\
\hline & $\checkmark$ & Objetivos y contenidos \\
\hline & $\checkmark$ & Materiales multimedia e interactivos \\
\hline & $\checkmark$ & Actividades de aprendizaje \\
\hline & $\checkmark$ & Evaluación del aprendizaje \\
\hline & $\checkmark$ & Tutorías \\
\hline \multirow{5}{*}{ Barnard \& Echols (2015) } & $\checkmark$ & Perfil del estudiante \\
\hline & $\checkmark$ & Contenidos temáticos \\
\hline & $\checkmark$ & Estrategias de aprendizaje \\
\hline & $\checkmark$ & Características de tecnología educativa utilizada \\
\hline & $\checkmark$ & Evaluación del aprendizaje \\
\hline \multirow{5}{*}{ Zapata (2015) } & $\checkmark$ & Objetivos y epítome \\
\hline & $\checkmark$ & Unidades \\
\hline & $\checkmark$ & Guías docentes y guías didácticas de las unidades \\
\hline & $\checkmark$ & Materiales para cada unidad \\
\hline & $\checkmark$ & Tipos y funciones de los profesores \\
\hline \multirow{4}{*}{ Boettcher \& Conrad (2016) } & $\checkmark$ & Objetivos y metas a alcanzar, \\
\hline & $\checkmark$ & Recursos didácticos. \\
\hline & $\checkmark$ & Acciones de aprendizaje. \\
\hline & $\checkmark$ & Estrategias de evaluación de aprendizaje. \\
\hline \multirow{7}{*}{ Rushby \& Surry (2016) } & $\checkmark$ & Datos curriculares \\
\hline & $\checkmark$ & Objetivos generales de aprendizaje \\
\hline & $\checkmark$ & Contenidos del programa \\
\hline & $\checkmark$ & Actividades del aprendizaje \\
\hline & $\checkmark$ & Fuentes de información \\
\hline & & Herramientas de comunicación y el uso de los medios \\
\hline & $\checkmark$ & Evaluación del aprendizaje \\
\hline
\end{tabular}

Fuente: Marciniak \& Sallán. (2017). Un modelo para la autoevaluación de la calidad de programas de educación universitaria virtual

Es importante destacar que no existe una receta genérica en cuanto a los elementos que debe contener un programa formativo virtual, es decir que no hay fórmulas o bosquejos para elaborar o diseñar un programa virtual; por consiguiente, cada programa es único, lo que hace pensar que su estructura y funcionamiento va a depender de los objetivos 
planteados, a quienes van dirigidos, los contenidos a impartir, los recursos con los que cuenta y puede ofrecer a los usuarios, etc.

Luego de realizar un análisis entre las directrices emitidas por HECQ (1997) y aquellas que, planteadas por algunos autores, se puede observar que existen algunos elementos que son comunes y que podrían servir como indicadores estándares para un programa formativo virtual. Entre los elementos de mayor frecuencia se pueden destacar los siguientes:

$\checkmark$ Objetivos y competencias de aprendizaje;

$\checkmark$ Estrategias de enseñanza;

$\checkmark$ Contenido temático;

$\checkmark$ Materiales y recursos didácticos;

$\checkmark$ Actividades de aprendizaje;

$\checkmark$ Estrategias de evaluación del aprendizaje.

\section{Resultados}

La educación Técnica y Tecnológica en Ecuador pasó a formar parte del sistema de educación superior en el año 1998, la primera evaluación fue realizada en el año 2007 y la última en el 2014, es decir que en 7 años los institutos estuvieron sujetos a 3 evaluaciones, lo que significa que en promedio fueron evaluados cada 28 meses. Las tres evaluaciones realizadas a los Institutos Superiores Técnicos y Tecnológicos tomaron como referencia diferente los criterios de evaluación, sin embargo, la frecuencia de uno de sus criterios refleja la importancia que este tiene en la calidad de la educación, tal es así que:

La primera evaluó 5 criterios: Docencia, Investigación, Vinculación con la comunidad y Gestión administrativa.

$\checkmark$ La segunda evaluó 3 criterios de: Docentes, Estudiantes y Administración.

$\checkmark$ La tercera evaluó 5 criterios: Pertinencia, Currículo, Calidad de la docencia, Infraestructura y Ambiente institucional. 
En las tres evaluaciones realizadas el criterio que ha estado presente en todas es el criterio relacionado a Docentes, lógicamente los docentes representan un pilar muy importante en la calidad de la educación que ofrece una institución a sus estudiantes y a la comunidad. El Modelo actual de evaluación de los Institutos Superiores Técnicos y Tecnológicos aplica el Método de Decisión Multicriterio (MDM), mismo que consta de 50 indicadores de los cuales 19 pertenecen al criterio Docencia lo que representa el 38\% del peso total de la evaluación. Es importante destacar que, de los 19 indicadores, 11 son de responsabilidad institucional en cuanto a los requisitos mínimos de contratación y los ocho restantes están bajo responsabilidad directa del docente en cuanto a la capacitación, formación y crecimiento profesional. En ninguna de las 3 evaluaciones realizadas hay un solo indicador que haga referencia a las competencias tecnológicas del docente, tomando en cuenta que las Tecnologías de la Información y Comunicación son tan necesarias en muchas actividades cotidianas y es ilógico pensar que en el modelo de evaluación no exista un indicador evalúe la cultura virtual de los ISTT.

En la formación de nivel técnica y tecnológica no existen ofertas educativas en cuanto a carreras virtuales se refiere, lo más cercano son las ofertas en modalidad a distancia, seguida de la modalidad semipresencial, siendo su fuerte la modalidad presencial, no se pudo evidenciar que algún instituto de sostenimiento particular, cofinanciado y mucho menos público este ofertando carreras en modalidad virtual.

\section{Conclusiones}

Los Institutos Superiores Técnicos y Tecnológicos no han incursionado en ampliar la oferta educativa en modalidades virtuales, no están aprovechando el uso de tecnología para llegar a un nicho de mercado educativo que por factor tiempo y/o distancia no acceden a la modalidad presencial del sistema de educación superior.

Al no contar con ofertas en modalidad virtual en el nivel técnico y tecnológico, es evidente que los docentes y estudiantes no han adquirido las destrezas tecnológicas requeridas para hacerle frente a una modalidad compleja, que, si bien brinda facilidades y 
ventajas rompiendo barreras de tiempo y espacio, los docentes no están preparados para los retos que exige la sociedad.

Los organismos de control en este caso el Consejo de Aseguramiento de la Calidad de la Educación Superior (CACES) no ha incluido en los criterios de evaluación ni en los indicadores, parámetros que permitan evaluar las competencias tecnológicas de los docentes, tomando en cuenta que hoy en día se requiere de tutores virtuales.

Los Institutos Superiores Técnicos y Tecnológicos no han desarrollado una cultura de calidad en función de los parámetros establecidos, solo se trata de cumplir las exigencias del CACES por obligación mas no por vocación, en este sentido al no existir indicadores sobre el aprendizaje virtual en este nivel de formación, no se han realizado los esfuerzos necesarios para promover la modalidad virtual como alternativa de accesibilidad y cubrir la demanda insatisfecha de los estudiantes. 


\section{Referencia bibliográfica}

Asamblea Constituyente del Ecuador (1998). Constitución de la República del Ecuador.

Barnard, D. \& Echols, J. (2015). The Anatomy of K-12 Online Programs: Practical Ideas and Guidelines. London: Rowman and Littlefield.

Boettcher, J. \& Conrad, R. (2016). The Online Teaching Survival Guide: Simple and Practical Pedagogical Tips. London: John Wiley and Sons.

CEAACES (2013). Informe General sobre la Evaluación, Acreditación y Categorización de las Universidades y Escuelas Politécnicas. Consejo Nacional de Evaluación, Acreditación y Aseguramiento de la Educación Superior.

CEAACES (2015). Metodología para la determinación de resultados del proceso de evaluación. En Informe Preliminar Informe Preliminar de la Evaluación Institucional. Consejo Nacional de Evaluación, Acreditación y Aseguramiento de la Educación Superior.

CONEA (2007a). Informe de evaluación externa del Instituto Tecnológico Superior Cordillera. Consejo Nacional de Evaluación y Acreditación de la Educación Superior Ecuatoriana (CONEA).

CONEA (2009a). Evaluación del Desempeño Institucional de los Institutos Superiores. Comisión de Institutos

Congreso Nacional del Ecuador (1977). Ley de Educación y Cultura. Decreto 1903. Registro Oficial $\mathrm{N}^{\circ} 461$. 
EducarPlus (2018). Lista de institutos técnicos y tecnológicos para postulaciones 2019 -2020. Recuperado de: https://educarplus.com/2018/07/lista-institutos-tecnicostecnologicos-postulaciones-snna-bachiller-2017-2018.html

Fainholc, B. (2004). La calidad en la educación a distancia continúa siendo un tema muy complejo. RED. Revista de educación a distancia, 12. Recuperado de http://revistas.um.es/red/article/view/25311/24591

García Aretio, L. (2014). Bases, mediaciones y futuro de la educación a distancia en la sociedad digital. Madrid, Síntesis.

García, L. (1998). Indicadores para la evaluación de la enseñanza en una universidad a distancia. RIED - Revista Iberoamericana de Educación a Distancia. 1(1), 63-86. Recuperado de http://ried.utpl.edu.ec/sites/default/files/files/pdf/v\%201-1/volumen1-1.pdf

HECQ (Higher Education Compliance and Quality). (1997). Guidelines for Distance Education. London: HECQ.

Mandato 14, Consejo Nacional de Evaluación y Acreditación de la Educación Superior Ecuatoriana (CONEA).

Marciniak, R. (2016). Autoevaluación de programas de educación universitaria virtual (Tesis doctoral). Universitat Autónoma de Barcelona: Barcelona.

Marciniak, R., \& Sallán, J. (2017). Un modelo para la autoevaluación de la calidad de programas de educación universitaria virtual. Revista de Educación a Distancia, (54).

Mery, Y. \& Newby, J. (2014). Online by Design: The Essentials of Creating Information Literacy Courses. Plymouth: Rowman y Littlefield. 
Meza, J. (2012). Modelo pedagógico para proyectos de formación virtual. Bonn: GIZ.

Ruiz, C. \& Dávila, A. (2016). Propuesta de buenas prácticas de educación virtual en el contexto universitario. RED. Revista de Educación a Distancia, 49(12). Recuperado de http://www.um.es/ead/red/49

Rushby, N. y Surry, D. 2016. Wiley Handbook of Learning Technology. New York, John Wiley and Sons.

Shattuck, K. (2014). Assuring Quality in Online Education: Practices and Processes at the Teaching, Resource, and Program Levels. Sterling: Stylus Publishing, LLC

SENESCYT-MINEDUC (2011). Acuerdo Interinstitucional N²011-002. Secretaría Nacional de Educación Superior, Ciencia, Tecnología e Innovación y Ministerio de Educación.

Zapata-Ros, M. (2015). Calidad en enseñanza abierta online universitaria: del aula virtual al MOOC. Campus Virtuales, 4(2), 86-107. Recuperado de https://issuu.com/revistacampusvirtuales/docs/7 